\title{
DER NEU ERSTARKTE UNDERGROUND. NEW YORK-HYPE UND US-AMERIKANISCHE INDEPENDENT-STRÖMUNGEN VORM HINTERGRUND DES 11. SEPTEMBERS
}

\section{Martin Büsser}

Die Ereignisse des 11. Septembers 2001 und deren politische Folgen haben in der US-amerikanischen Rock- und Popmusik, von der in diesem Text vorwiegend die Rede sein soll, ganz unterschiedliche Spuren hinterlassen. Zum einen kam es zu direkten Reaktionen, die - kurzfristig - von Zensurmaßnahmen bis zu groß angelegten Festivals und - langfristig - zu Songs reichten, in denen der 11. September und dessen Folgen thematisiert wurde. Diese Reaktionen fanden vorwiegend im so genannten Mainstream statt. Die Auswirkungen, die der 11. September zum anderen auf den Underground, vor allem auf die neu erstarkten, in den Medien seitdem auffällig präsenten subkulturellen Strömungen von New York hatte, lassen sich dagegen weniger direkt fassen, da kaum eine der Bands aus den (im weitesten Sinne) PostPunk-Szenen in ihren Songs direkt auf den 11. September Bezug nahm. Dass der 11. September und die auf inn folgende Welle des Patriotismus jedoch nachweislich zu einer Wiederbelebung des musikalischen Undergrounds in den USA beigetragen hat, der sich zudem explizit im Sinne einer Gegenkultur, eines "anderen Amerikas", präsentiert und sich unter anderem mittels der »Bands-Against-Bush «-Kampagne auch politisch positioniert hat, soll im Folgenden anhand einzelner Beispiele dargelegt werden. Die Zweiteilung dieses Textes folgt zudem der These, dass in den USA nicht nur die ökonomische Krise der Musikindustrie, sondern auch die im Zuge des 11. Septembers offen reaktionäre Politik der Bush-Regierung zu einer Trennung zwischen Underground und Mainstream geführt hat, wie sie in dieser Schärfe und Undurchlässigkeit seit den 1980er Jahren nicht mehr existiert hat.

Die Verwendung der Kategorien Underground und Mainstream ist stets problematisch, da eine solche Zweiteilung oft willkürlichen Kriterien unterliegt. Waren zum Beispiel Sonic Youth nur so lange Underground, wie sie auf 
Independent-Labels veröffentlichten, oder konnten sie auch noch als Band auf dem Major-Label Geffen aufgrund ihres unkonventionell ruppigen Sounds als Underground gelten? Wenn hier also dennoch von Underground und Mainstream die Rede ist, handelt es sich lediglich um Hilfsbegriffe, die zumindest in dem Maße nicht bloß willkürlichen Geschmacksurteilen unterliegen, in dem Bands in jüngerer Zeit selbst wieder ganz bewusst Trennungslinien ziehen und ihre eigene Underground-Positionierung bis hin zur musikalischen wie ökonomischen Selbstmarginalisierung aktiv vorantreiben.

Diesbezüglich lässt sich in den USA seit zwei bis drei Jahren eine Entwicklung konstatieren, die entgegen der einst als »Mainstream der Minderheiten « (Holert/Terkessidis 1995) bezeichneten Synergieeffekte zwischen subkulturellen Strömungen und Musikindustrie verläuft. »Mainstream der Minderheiten « bezog sich Anfang der 1990er Jahre unter anderem auf das im Zuge von Nirvana und des Grunge-Booms neu erstarkte Interesse ${ }^{1}$ der Major-Labels, Bands aus dem so genannten Underground, die bislang auf lokalen Indie-Labels veröffentlicht hatten, unter Vertrag zu nehmen. Das führte dazu, dass die Mainstream-Underground-, also auch die MehrheitMinderheit-Dichotomie aufgrund einer neu entstandenen Durchlässigkeit nicht mehr aufrecht zu erhalten war. Vieles spricht allerdings dafür, dass die wirtschaftliche Krise, in der sich die großen Plattenfirmen derzeit befinden, und die politische Situation in den USA dem gegenüber für eine neue Polarisierung gesorgt haben, die sich musikalisch, aber auch in der Wahl ganz bestimmter Distributionswege ausdrückt. Auf die aus ökonomischen Gründen zurückgegangene Risikobereitschaft der großen Firmen, Musik jenseits sicherer Banken wie den (international erfolgreichen) "Superstar «Formaten unter Vertrag zu nehmen, reagierten Klein- und Kleinstlabels mit musikalisch immer höherer Risikobereitschaft, wohl wissend, dass der Markt für kompromisslose musikalische Ansätze in Zeiten der Unzufriedenheit weder sinkt noch wegbricht, sondern eher sogar ansteigt.

1 Neu erstarkt insofern, als dass die Musikindustrie schon einmal, während der Blütezeit des Punk Mitte der 1970er Jahre, sehr schnell auf eine neu entstandene Subkultur reagiert hatte: Zahlreiche frühe Punkbands, darunter The Sex Pistols, The Clash, The Damned und The Ramones veröffentlichten ihre Platten auf Major-Labels. 


\section{Was die Welt mitbekam...}

Zu den direkten Auswirkungen des 11. Septembers zählte zunächst einmal die schnell erstellte, an die Radiostationen des Clear Channel-Imperiums gerichtete Liste von Songs (vgl. S. 60f.), die vorübergehend nicht mehr gespielt werden sollten, wobei es sich um einen Index handelte, der auf den ersten Blick (im Gegensatz zum ersten Golfkrieg, während dessen Verlauf von US-amerikanischen Radiostationen keine Antikriegslieder gespielt wurden) nicht offenkundig als politische Zensur daherkam, sondern der angeblich aus Pietät gegenüber den Opfern des 11. Septembers erstellt worden war. Hierzu zählte beispielsweise Geri Halliwells Song »It's Raining Men«, dessen eher harmloser, der Redewendung »Es regnet Hunde und Katzen « entlehnter Refrain, nun auch mit jenen Menschen assoziiert werden konnte, die sich aus den Fenstern des brennenden World Trade Centers gestürzt hatten. Betroffen waren unter anderem auch »Another One Bites The Dust« von Queen und »Knocking On Heaven's Door« in all seinen verschiedenen Einspielungen, aber auch »Imagine « von John Lennon, ein Song, dessen Zeile »Imagine there's no heaven « (bzw. deren atheistische Implikation) als Beleidigung der Opfer des 11. Septembers angesehen wurde. Vielsagend und der Behauptung widersprechend, dass es sich diesmal nicht um politische Zensur handelte, war die Indizierung des kompletten Oeuvres von Cat Stevens, weil dieser bereits vor Jahren zum Islam konvertiert war.

Auf den 11. September folgten schnell organisierte und weltweit in Fernsehen und Radio übertragene New Yorker Großkonzerte, schließlich auch erste Songs, in denen die Terroranschläge thematisiert wurden, darunter »Let's Roll« von Neil Young, veröffentlicht auf dessen CD Are You Passionate. Das Stück ist eine Hymne auf jene Passagiere, die das vierte entführte Flugzeug am 11. September zum Absturz gebracht hatten, und fordert mit seinem Refrain »Let's Roll« dazu auf, nun in den Krieg zu ziehen. Bruce Springsteen bemühte sich dagegen auf seinem nach Jahren wieder einmal mit der E-Street Band eingespielten Album The Rising darum, einen möglichst unparteiischen Blickwinkel einzunehmen und gab jenen Septembertag unter anderem aus der Sicht eines Feuerwehrmanns und eines Selbstmordattentäters wieder.

Ein Blick auf die musikalische Verarbeitung des 11. Septembers wäre nicht vollständig, wenn man nicht auch die Rezeption der politischen Folgen berücksichtigen würde, darunter die Militäreinsätze in Afghanistan und im Irak, aber auch die unter der Bezeichnung »Patriotic Act zusammengefassten inneramerikanischen Vorkehrungen zur Terrorbekämpfung, die 
einschneidende Eingriffe in die Freiheiten der Bürger zur Folge hatten, begleitet von einer massiven, patriotischen Kriegsrhetorik seitens der BushRegierung, einer neuen Einteilung der Welt in »good« und »evil«.

Es wäre verwunderlich, wenn all dies spurlos an der Popmusik vorbeigegangen wäre. Sieht man jedoch einmal von zahlreichen, zum Teil erfolgreichen Songs $a b$, die auf der Patriotismus-Welle mitgeschwommen waren (darunter das kriegerische "Courtesy Of The Red, White And Blue des Countrysängers Toby Keith), aber auch von der weitaus geringeren Zahl kritischer Stimmen gegenüber der politischen Linie von George W. Bush (darunter George Michaels Video zu "Shoot The Dog«, ein Cartoon, der unter anderem den britischen Premierminister Tony Blair als speichelleckenden Schoßhund von George W. Bush darstellt und inn am Ende wie "Dr. Seltsam« aus dem Film von Stanley Kubrick auf einer Bombe reiten lässt), haben der 11. September und seine politischen Folgen keine weit reichenden Spuren im kommerziellen Popbetrieb hinterlassen. Dass es immer wieder die selben Beispiele von Neil Young über Bruce Springsteen bis zu Paul McCartney (»Freedom«) und George Michael waren, die in diesem Zusammenhang zitiert wurden, belegt geradezu, dass innerhalb des Pop-Mainstreams keine grundlegende Abweichung vom Tagesgeschäft und den bewährten Erfolgskursen zu erkennen war.

Es gelte aufzuzeigen, schreibt Oliver Tolmein (2002: 9) im Vorwort zu seinem Buch Vom deutschen Herbst zum 11. September, dass sich keine im Zusammenhang mit dem 11. September geäußerte Prognose so wenig bewahrheitet habe wie jene, dass »nichts mehr so sei, wie es bisher war « dies gilt auch, wenn nicht sogar in besonderem Maße, für den weiteren Verlauf, den der Pop-Mainstream genommen hat. Es kam mitnichten zu einem »Ende der Spaßgesellschaft«, für das Peter Scholl-Latour unmittelbar nach dem 11. September während eines Fernsehauftritts plädiert hatte im Gegenteil: Das Thema, das die deutschen Medien neben dem Ausbruch des Irakkrieges am meisten beschäftigte, war der Casting-Wettbewerb »Deutschland sucht den Superstar«. Dieses Nebeneinander von politischen Ereignissen, die mit Bürgerprotesten einhergingen, und dem massenhaften, davon völlig losgelösten Interesse für politikfernen Pop spricht vielmehr für eine Kontinuität des Apolitischen, geradezu Ahistorisch-Redundanten innerhalb des kommerziellen Popbetriebs. Allenfalls ließe sich die These aufstellen, dass politische Krisenzeiten die Attraktivität von Medienspektakeln wie dem »Superstar«-Wettbewerb erhöhen. 


\section{»Dance to the Underground«}

Wie aber reagierte der Underground, jenes Netzwerk aus zahlreichen Independent-Labels, dessen Aktivitäten nicht unmittelbar von Zensur betroffen sind und das deshalb viel problemloser, also auch schneller, unabhängig von zahlreichen korrektiven Instanzen wie Management, Radiostationen oder TV-Videokanälen, auf politische Ereignisse regieren kann? Dort, wo beispielsweise Bands und Labelbetreiber miteinander identisch sind, bedurfte es keiner größeren Absprachen, konnten zum Beispiel direkt persönliche politische Statements auf die eigene Website gesetzt werden. In den ersten Monaten nach dem 11. September konnte allerdings von einer direkten, aktiven Reaktion noch keine Rede sein, denn zumindest in New York sahen sich die meisten Bands erst einmal mit einer ganz neuen Situation, nämlich mit einem bislang nicht gekannten Medieninteresse konfrontiert. Die internationale Presse stürzte sich geradezu gierig auf das subkulturelle Leben von New York, als gelte es, anhand von kleinen Bands, die vorwiegend in den Clubs von Williamsburg, dem neuen Zentrum der Boheme, auftraten, die kulturelle Vielfalt dieser so stark auf den Prüfstand gestellten Stadt demonstrativ hervorzuheben und damit implizit darauf hinzuweisen, dass neben dem Hurra-Patriotismus auch - und zwar vor allem in New York - so etwas wie ein »anderes « Amerika existiert - dessen künstlerische Kreativität und Unabhängigkeit wiederum als Beweise für US-amerikanische Grundwerte wie Freiheit und Demokratie herangezogen, wenn nicht gar instrumentalisiert werden konnten.

Beflügelt wurde dieser Blick auf New York nicht zuletzt durch den enormen, unerwarteten Erfolg der Strokes, deren umjubeltes LP-Debüt Is This It im Sommer 2001 erschienen war. Nicht ohne eine Spur Zynismus lässt sich behaupten, dass zwar der Hype um die Strokes und deren einfachen Garagen-Rock in (typisch New Yorker) Velvet-Underground-Tradition bereits vor dem 11. September ansetzte und so auch unabhängig von den politischen Ereignissen einen generellen New-York-Boom nach sich gezogen hätte, dass aber der 11. September diese neue Begeisterung in hohem Maße verstärkt, beschleunigt und emotional aufgeladen hat. Mike Evans schreibt in seinem Buch New York City Rock:

"Es bleibt eine offene Frage, ob die Renaissance des NY-Rock, die mit den Strokes eingeleitet wurde, einen Teil des Heilungsprozesses nach den Anschlägen darstellt. Es gibt zumindest eine Parallele zu der oft geäußerten These, dass sich die Begeisterung, mit der die Beatles 1964 von den Amerikanern empfangen wurden, als eine therapeutische Nachwirkung auf 
die Ermordung John F. Kennedys im November 1963 erklären lässt« (Evans 2003: 13).

$\mathrm{Ob}$ es einen solchen direkten Zusammenhang gegeben hat, eine Art therapeutische Solidaritätsbekundung mit dem subkulturellen Leben dieser Metropole, wird sich nie eindeutig beantworten lassen. Fest steht jedoch, dass plötzlich nahezu jede New Yorker Band, die gerade mal eine Single auf dem Markt gebracht hatte (z.B. The Yeah Yeah Yeahs) und die im weitesten Sinne nach dem unbedarften, manchmal auch unbeholfenen Flair von Proberaum klang, von der internationalen Presse als Bestandteil eines großen, New Yorker Underground-Revivals gefeiert wurde - ein Hype, der insofern eine therapeutische Lesart anbietet, als die mit dieser Musik wieder entdeckte Einfachheit und Direktheit ganz den, nach dem 11. September proklamierten Forderungen entsprach, man müsse sich wieder auf das Wesentliche besinnen.

Die Presse begann ab Ende 2001 so euphorisch von der neuen RockMetropole zu sprechen, wie dies seit den späten 1970er Jahren, der New Yorker Blütezeit von Punk und New Wave (The Ramones, Blondie, The Talking Heads, Patti Smith u.v.m.) nicht mehr der Fall war - und das, obgleich sich die New Yorker Subkultur 2001 gar nicht in einem Aufschwung, sondern eher sogar in einer Krise befunden hatte. Die hohen Mietpreise des ehemaligen subkulturellen Zentrums Greenwich Village hatten für eine Abwanderung von Clubs und Proberäumen zuerst nach Brooklyn, schließlich sogar in die Bronx gesorgt, also die Aktivitäten der Subkultur immer weiter aus dem geographischen Zentrum verdrängt. Unter Bürgermeister Giuliani war es zudem zur Wiedereinführung des »Cabaret Law« gekommen, eines Gesetzes, das den Clubs verbot, ohne spezielle Lizenz Tanzveranstaltungen abzuhalten, was zu einer Beschneidung der gesamten Musikkultur geführt hatte, auf welche die funkig-tanzbare New Yorker Punk- und Wave-Band Radio 4 mit ihrem Song »Dance To The Underground « reagierte. »Wenn du diese >Cabaret Licence nicht hast «, erklärten die Musiker in einem Interview (zit. n. Stalling 2003: 114), »darfst du den Leuten in deinem Laden nicht erlauben zu tanzen. Und die ist hart zu kriegen.«

Die neue Underground-Metropole war also nicht zuletzt ein mediales Konstrukt: Junge, unkonventionelle Bands wie Interpol, The Moldy Peaches, The Rapture, Black Dice und The Liars, um nur einige Namen zu nennen, hätte man in einer Großstadt wie New York jederzeit finden können, ohne dass dies ein Grund dafür gewesen wäre, von der »Hauptstadt des Rock'n' Roll« (Evans 2003: 206) zu sprechen. Doch nicht zuletzt der Schock des 11. Septembers dürfte dazu geführt haben, dass die von diesen Bands vertretenen Werte (das Grundethos von Independent und »Do It Yourself«, die 
bevorzugte, kommunikativ intime Situation der kleinen Clubs und Musik als Ausdruck elementarer Gefühle jenseits von Stargehabe) als Ausdruck eines Zusammenhalts empfunden wurden, welcher der allgemeinen Betroffenheit und dem Wunsch nach einem Neuanfang entgegen kam. So schrieb beispielsweise Ethan Brown im New York Magazine mit deutlichem Verweis auf den 11. September:

"Warum ist die Musik von New York wieder so bedeutend? Weil es sich um die lebendigste und vielseitigste Szene handelt, die diese Stadt seit den frühen Achtzigern erlebt hat. Weil sie Brooklyn zu einem coolen Ort gemacht hat. Weil sie das Licht am Ende dieses langen, schwarzen Tunnels der zynischen Neunziger darstellt. Weil sie die Schmerzen lindert, die der letzte Herbst mit sich gebracht hatte« (Ethan Brown, zit. n. Evans 2003: 206).

Was die Presse nach dem 11. September in New York vorfand, war keine Szene, die sich lautstark politisch positionierte, deren musikalisches Selbstverständnis dennoch bereits einen Wertewandel ankündigte: Die Art und Weise, wie sich Musiker hier artikulierten, ihr Umgang mit dem Publikum und auch ihr Engagement, sich gegenseitig unterstützende Netzwerke jenseits der Musikindustrie aufzubauen, zeugten von einem respektvollen Umgang miteinander, der im Schatten des 11. September fast schon als humanistisch gefärbte Gegenhaltung zur vollmundigen Kriegsrhetorik gelesen werden konnte.

Ein gutes Beispiel hierfür sind die aus der New Yorker »Antifolk«-Szene hervorgegangenen Moldy Peaches, deren auf dem Londoner Rough-TradeLabel erschienenes Debüt zufälligerweise am 11. September 2001 in die Läden kam. Die Platte enthält (ein weiterer seltsamer Zufall) unter anderem den Song »New York City Is Like A Graveyard«, in dem die Wolkenkratzer der Stadt mit Grabsteinen verglichen werden und dessen Zeilen »All the yuppies getting married / All the yuppies getting buried « nachträglich einen ebenso bitteren wie zynischen Beigeschmack erhielten. Was die Band aber eigentlich auszeichnete, waren ihr liebreizender Hang zum Dilettantismus und ihre entwaffnend selbstironischen Liveauftritte, während derer die Musiker (angeführt von Sängerin Kimya Dawson und Sänger Adam Green) beispielsweise auf der Bühne in Lachkrämpfe ausbrachen, wenn sie wieder einmal ihren Text vergessen hatten. Desillusionierende Zeilen wie »| wanna be a hippie, but I forgot how to love (»Who's Got The Crack«) wurden in eine Musik gepackt, deren Charme (etwa der Einsatz einer beherzt amateurhaft gespielten Blockflöte) und gespielte Niedlichkeit durchaus kathartische Wirkung hatten. Als die Moldy Peaches in ihren seltsamen Häschen- und Einhorn-Kostümen im Frühjahr 2002 auch auf deutschen Bühnen standen, 
ließen sie es sich nicht nehmen, die Gäste im Publikum nach ihren Konzerten ausnahmslos zu umarmen - eine symbolische Geste, die zu jener Zeit gar nicht anders als politisch gelesen werden konnte.

Treffpunkt und musikalischer Austragungsort der »Antifolk«-Szene, sind die jeden Montag im New Yorker Sidewalk Café stattfindenden »Open Mic Sessions«. In Abgrenzung zum konventionellen, von den Protagonisten als bieder empfundenen Folk, zählt hier die Freiheit des Ausdrucks mehr als technisches Können. Die nonkonformistische Haltung der Szene drückt sich zudem nicht in herkömmlichen Protestsongs aus, sondern unter anderem dadurch, dass hier musikalisch bewusst eine kleine Form gewählt wurde, vorgetragen mit einem oft schäbigen Equipment, das bereits signalisiert, dass man selbst nicht zu den Siegern des Systems gehört.

Für die Szene gilt, was in diesem Text bereits gegenüber anderen Phänomenen der New Yorker Subkultur festgestellt wurde: Sie entstand nicht als Folge des 11. Septembers - die »Open Mic Sessions « unter »Antifolk«Banner finden nun schon seit mehr als fünfzehn Jahren im Sidewalk Café statt -, und wäre aufgrund ganz bestimmter Konstellationen (die Strokes haben sich als große Fans und Förderer der Moldy Peaches hervorgetan) wohl auch ohne die Ereignisse des 11. Septembers von den Medien entdeckt und gefeiert worden. Durch den 11. September und seine Folgen erhielt die Berichterstattung jedoch eine zum Teil geradezu ehrwürdig sakrale Note und betonte zugleich immer wieder die politische Bedeutung dieser nur vermeintlich apolitischen Musik. Über einen »Antifolk«-Abend im Frankfurter Dreikönigskeller war beispielsweise in der Frankfurter Rundschau zu lesen:

»Die Sängerinnen und Sänger des Antifolk vermitteln Gefühl und Wärme, sie stehen als Menschen auf der Bühne, nicht als Stars. In ihren witzigsten Momenten zaubern sie ein Lächeln auf die Gesichter der Zuhörer. Unmittelbare politische Aussagen spielen keine große Rolle. Über die Haltung muss man sich nicht erst verständigen. Dass man zum anderen Amerika gehört und nicht etwa zu den Befürwortern des Irak-Feldzugs, ist ohnehin klar« (Michalzik 2002).

Ein Rezensent der FAZ sah darin sogar eine Verweigerungshaltung im Sinne Adornos am Werk:

»Wie im Einsatz von Xylophonen, Blockflöten, Tröten und Triangeln artikuliert sich darin die Weigerung, sich einem Kulturbetrieb anzudienen, in dem Erwachsensein, wie schon Adorno wußte, oft nur ein anderes Wort für Regression ist. [...] Womöglich hätte der schärfste Kritiker populärer Musik sich von den Moldy Peaches bekehren lassen« (Klaue 2002). 
An dieser Stelle könnte eingewendet werden, dass ein solcher, seitens der Presse eingeräumter politischer Subtext nicht unmittelbar mit den politischen Ereignissen seit dem 11. September zusammenhängt, sondern ohnehin Bestandteil eines schon lange ausgebildeten und vor allem in Deutschland verbreiteten popjournalistischen Jargons ist, die jeweils bevorzugte Musik als politisch dissident aufzuwerten. Unabhängig von der Frage, inwieweit die Medien an einem Bild der Repolitisierung des neu erstarkten (oder eben, genauer gesagt, erstmals wieder stärker beachteten) musikalischen Undergrounds in den USA gearbeitet haben, haben die verschiedensten Independent-Segmente tatsächlich eine politische Positionierung vorgenommen. Diese steht jedoch - nicht nur inhaltlich - in einem deutlichen Gegensatz zu offenkundigen Statements wie dem »Let's Roll«-Song von Neil Young. Independent und subkultureller Zusammenhalt selbst, aber auch eine musikimmanente Verweigerungshaltung erlebten eine auf verschiedenste Weise politisch konnotierte Renaissance, nicht aber der klassische Protestsong.

\section{»Mehr als Eminem und Michael Jordan...«}

Protest muss nicht notwendig vollmundig aus den Songs selbst heraus sprechen, wie viele Beispiele aus dem letzten Jahr gezeigt haben. Er kann auch über die strukturelle Ebene verbreitet werden, über außermusikalische Netzwerke oder über einen, der Musik beigegebenen Subtext. Ein klassisches Beispiel für eine primär strukturell arbeitende Band sind Fugazi aus Washington DC. Sie gelten vielen bis heute als vorbildlich, wenn es darum geht, das Independent-Ethos im Sinne einer politischen Strategie zu nutzen. Das »Do it yourself«-Prinzip der Independent-Labels ist selbst noch kein Ausdruck politischen Dagegens, sondern verkörpert lediglich den alten USamerikanischen Wahlspruch, dass ein jeder ganz allein für seinen Erfolg auf dem Markt verantwortlich ist. Fugazi haben daraus jedoch ein Politikum gemacht, indem sie nicht Einzelkämpfertum, sondern Vernetzung von Kräften in den Mittelpunkt stellten. Die Band weigert sich seit nunmehr fünfzehn Jahren, mit der Musikindustrie zusammenzuarbeiten, bestimmt Auftrittsorte und Eintrittspreise weitgehend selbst, schaltet keine Anzeigen in kommerziellen Magazinen und tritt regelmäßig auf Benefizkonzerten - etwa im Rahmen der Seattle-Proteste - auf. Mit all diesen Entscheidungen trugen Fugazi dazu bei, dass sie gar nicht anders als politisch wahrgenommen werden können. Ihre Haltung ist bis heute unmissverständlich, obwohl sie keine dezidiert politische Nummer in ihrem Repertoire haben. Zeilen wie »I am a patient boy / I wait, I wait, I wait « aus ihrem bekanntesten Song »Waiting 
Room« bleiben völlig offen, drücken höchstens ein allgemeines Unbehagen aus. Während eine Band wie die Toten Hosen offen antifaschistische Songs in ihrem Repertoire hat und dennoch nicht verhindern kann, dass sich auch Böhse-Onkelz-Fans im Publikum befinden, ist es Fugazi aufgrund zahlreicher außerhalb der Musik getroffener Entscheidungen gelungen, allen falschen Vereinnahmungen vorzubeugen.

Die »Bands Against Bush«-Kampagne, die vorm Hintergrund des Irakkrieges ihre flächendeckende Verbreitung über die ganze USA fand, hat einen ähnlichen Ansatz gewählt. Von Portland über New York bis Olympia und Los Angeles sind die einzelnen lokalen »Bands Against Bush «-Gruppen mit je eigener Homepage im Internet vertreten. Neben dem Bereitstellen und Sammeln von Internet-Links zu politischen Organisationen verfolgt die Initiative vor allem die Taktik, die unterschiedlichsten Bands für Konzerte zu gewinnen, also Kräfte zu bündeln, ganz gleich, ob die jeweilige Band explizit politische Nummern im Programm hat oder nicht. Namen wie Le Tigre, Sonic Youth, The Liars, The Locust und Erase Errata, die »Bands Against Bush « bereits unterstützt haben, machen deutlich, dass es hier nicht um klassischen Agit-Prop geht, sondern darum, den in den letzten Jahren allzu leichtfertig gebrauchten »Alternative«-Begriff an politische Selbstverständlichkeiten rückzubinden. Gegenkultur, die in den USA lange Zeit fast ausschließlich als ästhetische Abgrenzung gegenüber dem Mainstream angesehen wurde, verortet sich mit Hilfe der Kampagne erstmals wieder politisch, ohne jedoch auf ästhetischer Ebene belehrend oder doktrinär daherzukommen. Das Politische bleibt hier also selbst dann, wenn eine Instrumentalband ganz ohne eindeutige Botschaften auftritt, über den Rahmen präsent.

Ein weiteres subkulturelles Netzwerk, das Independent-HipHop-Label »Anticon«, versammelt ebenfalls bis auf wenige Ausnahmen Künstler, die in ihren Songs nur selten dezidiert Stellung zur politischen Lage beziehen, sondern in erster Linie an einer musikalischen Abgrenzung gegenüber dem Mainstream-HipHop arbeiten (»Anticon«-Künstler wie cLOUDDEAD, Why? und Deep Puddle Dynamics klingen beispielsweise bewusst androgyn, wenden sich gegen jegliche Macho-Attitude). Indem ein Link auf der Label-Homepage zu Noam Chomsky führt, findet eine Positionierung statt, die es den Musikern zugleich ermöglicht, ihre eigene Arbeit von »Preaching « freizuhalten.

Ähnlich verbindlich, aber jenseits jeglicher Gesinnungsmusik arbeitete (der in diesem Fall britische Musiker) Matthew Herbert auf Goodbye Swingtime, dem vielleicht komplexesten politischen Album aus dem Jahre 2003. Die mit zahlreichen, hochkarätigen Gastmusikern eingespielte, an die 
Swing-Ära anknüpfende Big-Band-Platte verzichtet ebenfalls auf die klassische Form des Protestsongs und verlagert einen Großteil der politischen Aussage ins Klangmaterial. Mal sind die Geräusche eines Nadeldruckers zu hören, mit dem Herbert gerade eine Anti-Globalisierungs-Seite aus dem Internet ausdruckt, an anderer Stelle verarbeitet Herbert die Maschinengeräusche seiner Druckerei, um in klassisch marxistischer Intention jenen Produktionsprozess hörbar zu machen, der beim Entstehen kultureller Produkte wie Büchern und Schallplatten meist ausgeblendet wird. Auf Goodbye Swingtime findet sich das Politische nicht im Mittelpunkt der Musik. Es ist deren durch Samples, Zitate und auf der Hülle abgedruckte Buchempfehlungen mitgelieferter Subtext.

Zusammenfassend lässt sich feststellen, dass zahlreiche Faktoren zu einem Underground-Boom geführt haben, darunter ökonomische (die Abkehr von einer immer stärker auf sichere Mainstream-Acts setzenden Musikindustrie) und politische, aber auch der bis heute anhaltende New-YorkHype, dessen proklamiertes »Zurück zur Basis« mit der Reaktion auf den 11. September korrespondiert, sich wieder auf elementare Grundwerte zu besinnen. Die zahlreichen Netzwerke aus Independent-Labels, Clubs und Initiativen verstehen sich jedoch nicht als offenkundige Plattform für politischen Protest, vielmehr drückt sich die politische Selbstpositionierung wieder verstärkt im Independent-Ethos selbst aus, das auf ein »anderes «, gegenkulturelles und humanistisch geprägtes Amerika verweist, wie es Dose One, Mitbegründer des »Anticon«-Labels in einem Interview mit dem bayrischen »Zündfunk« 2003 formulierte: »Aus den USA kommen auch sehr schöne Dinge, also nicht nur Michael Jordan oder Eminem. «

\section{Literatur}

Evans, Mike (2003). New York City Rock. Mainz: Ventil Verlag.

Holert, Tom / Terkessidis, Mark (Hg.) (1995). Mainstream der Minderheiten. Pop in der Kontrollgesellschaft. Berlin: Edition ID-Archiv.

Klaue, Magnus (2002). „Wie ernst ist die Heiterkeit? Die Narren des Folkpop. «In: Frankfurter Allgemeine Zeitung vom 30. November, S. 41.

Michalzik, Stefan (2002). "Auch lieb. Antifolk auf Tour.« In: Frankfurter Rundschau, Nr. 288 vom 11. Dezember, S. 29.

Stalling, Tobias (2003). »Nach Punk in die Disco. Von der Pop Group zu Radio 4. Die Rückkehr des Post-Punk als Politikum.«In: Linke Mythen. Hg. v. Martin Büsser u.a. (= testcard - Beiträge zur Popgeschichte 12). Mainz: Ventil Verlag, S. 110-115.

Tolmein, Oliver (2002). Vom Deutschen Herbst zum 11. September. Die RAF, der Terrorismus und der Staat. Hamburg: Konkret Literatur Verlag. 


\begin{abstract}
September 11th and the new wave of patriotism caused no fundamental change in the development and aesthetics of mainstream-pop. The record industry focussed on risk-free and apolitical casting-acts like "Superstars" as a consequence of its financial crisis. At the same time there has been a boom of US-American independent-music since 2001. The international media hype of the $\gg$ New York underground « might be read as a declaration of solidarity with New York after 9/11, but also as a proclamation of a renaissance of (human and artistic) basics. Besides the hype there was, in fact, a comeback of independent- and do-it-yourself-values, but most of the contemporary independent musicians in the US don't write protestsongs anymore. They get connected with campaigns like »Bands against Bush«, but aren't interested in using their music as a kind of one-dimensional political statement.
\end{abstract}

